\title{
Two-Stage Load Shedding for Secondary Control in Hierarchical Operation of Islanded
} Microgrids

\author{
Zhou, Quan; Li, Zhiyi; Wu, Qiuwei; Shahidehpour, Mohammad
}

Published in:

IEEE Transactions on Smart Grid

Link to article, DOI:

10.1109/TSG.2018.2817738

Publication date:

2018

Document Version

Peer reviewed version

Link back to DTU Orbit

Citation (APA):

Zhou, Q., Li, Z., Wu, Q., \& Shahidehpour, M. (2018). Two-Stage Load Shedding for Secondary Control in Hierarchical Operation of Islanded Microgrids. IEEE Transactions on Smart Grid, 10(3), 3103 - 3111. https://doi.org/10.1109/TSG.2018.2817738

\section{General rights}

Copyright and moral rights for the publications made accessible in the public portal are retained by the authors and/or other copyright owners and it is a condition of accessing publications that users recognise and abide by the legal requirements associated with these rights.

- Users may download and print one copy of any publication from the public portal for the purpose of private study or research.

- You may not further distribute the material or use it for any profit-making activity or commercial gain

- You may freely distribute the URL identifying the publication in the public portal 


\title{
Two-Stage Load Shedding for Secondary Control in Hierarchical Operation of Islanded Microgrids
}

\author{
Quan Zhou, Member, IEEE, Zhiyi Li, Member, IEEE, Qiuwei Wu, Senior Member, IEEE and \\ Mohammad Shahidehpour, Fellow, IEEE
}

\begin{abstract}
A two-stage load shedding scheme is presented to cope with the severe power deficit caused by microgrid islanding. Coordinated with the fast response of inverter-based distributed energy resources (DERs), load shedding at each stage and the resulting power flow redistribution are estimated. The first stage of load shedding will cease rapid frequency decline in which the measured frequency deviation is employed to guide the load shedding level and process. Once a new steady-state is reached, the second stage is activated, which performs load shedding according to the priorities of loads. The effectiveness of the proposed scheme is verified through time-domain simulation in PSCAD/EMTDC based on a scaled-down microgrid system.
\end{abstract}

Index Terms-Microgrid islanding, secondary control, controllable loads, renewable energy resources, load shedding.

\section{INTRODUCTION}

$\mathrm{M}$ ICROGRIDS can operate in grid-connected and island modes to ensure the continuous supply of power [1]-[6]. In island mode, a microgrid will operate as a self-controlled entity to regulate its frequency and voltage by dispatching distributed energy resources (DERs) including wind turbine generator (WTG), photovoltaic (PV), battery energy storage system (BESS), and controllable loads [7], [8]. Accordingly, when the microgrid load is larger than its total generation capacity, the power outputs of DERs will increase to supply the local load demand and the frequency will drop rapidly due to the low effective inertia. In such circumstances, an appropriate load shedding procedure is inevitable. Otherwise, the microgrid would face significant transients and a complete collapse. In such circumstances, the immediate power deficit is determined by the power flow measurement at point of common coupling (PCC) which could be mitigated by a combination of microgrid options including fast responses of local generator units and load shedding. So the power generation deficit should be detected and taken into account in the load shedding scheme.

Due to low inertia of DERs, the power deficit caused by islanding could lead to rapid decline in the microgrid frequency, especially when the DER capacity is less than that of load. In such scenarios, under-frequency load shedding (UFLS) can stabilize the islanded microgrid to prevent the microgrid system collapse [9]-[12]. The conventional UFLS schemes are widely applied in power utilities due to its simplified design. Its settings are predefined, including frequency threshold values, percentage of shedding at each step, delay time, etc. Since they fail to estimate the actual power deficiency at each step, these load shedding schemes could result in excessive levels of load curtailment under different operating conditions [13]. Depending on frequency derivative measures to estimate power imbalances, adaptive load shedding schemes have been introduced in industry [14]-[16]. By continuously monitoring the rate of frequency change in the equivalent center of inertia, a procedure is proposed to adapt the shedding at each step to the primary frequency control response [17]. However, the oscillatory nature of frequency derivatives may pose misleading instantaneous measures.

In microgrids, due to presumed delays in islanding detections, it is often difficult to guarantee that frequency derivatives are measured close to the moment when the islanding occurs. To avoid error measurements caused by frequency derivative transients, adaptive UFLS schemes have been further improved by using frequency derivatives close to the center of inertia or calculating the average value, which is established by monitoring frequency response over periods of several hundred milliseconds [9]-[11]. However, delays are considered unacceptable for islanded microgrids facing severe power deficits and rapid frequency drops.

Although the system frequency often drops very rapidly when microgrid is facing severe disturbances which influence resources both inside and outside microgrid boundaries, some inverter-based DERs, such as WTGs with a BESS, can have a very fast primary control response to sustain the microgrid system frequency after disturbances [10],[18]-[20]. When facing severe under-frequency conditions, the primary control response depends largely on maximum capacities of these DERs rather than their response speed.

This paper focuses on circumstances when a microgrid is suddenly disconnected from the utility grid. In such circumstances, depending on the state of microgrid DERs, a proper level of loads should potentially be curtailed quickly in order to restore the microgrid system frequency before any system collapse [21]. The load shedding level and the related power flow redistribution can be estimated by considering the fast primary frequency control response of inverter-based DERs. Hence, the load shedding scheme must be coordinated with the corresponding DER characteristics for achieving the stated objectives of speed and quantity. Accordingly, we propose two stages in the proposed load shedding scheme.

The first stage will quickly stabilize the microgrid system frequency. The proposed scheme will consider the location and the quantity of load shedding for optimizing the system dynamics [22],[23]. Accordingly, the frequency deviation is used to select loads that will participate at the first stage of load shedding [24]. Then the estimated shedding will determine relative frequency deviations and redistributed power flow. The second stage is designed to restore the steady state frequency considering the supply priorities of loads [25]. This stage provides an adequate frequency margin for implementing the secondary control in a microgrid.

The main contributions of the paper are listed as follows:

(1) Proper load shedding is designed at two stages considering the curtailment allocation which is adapted to the prevailing condition in a microgrid. 
(2) The two-stage scheme considers coordinations among curtailable loads and DER responses for frequency restoration. (3) The relationship between the frequency deviation and the integral of active power deficit is considered for selecting the load curtailment locations and quantities.

The remainder of this paper is organized as follows. Section II discusses the steady state frequency decline and recovery based on the combined droop characteristics. Section III illustrates the relation between locally measured frequency deviations and the integral of local power deficits. A two-stage load shedding scheme is presented in Section IV. Case studies are presented in Section V to verify the effectiveness of the proposed scheme. Section VI concludes the paper.

\section{FREQUENCY RESPONSE OF AN ISLANDED MiCROGRID}

In island mode, the active power deficit can be reduced by either increasing the DER generation by primary frequency control or decreasing the system load demand by load shedding. Load shedding is regarded as an active power compensation that can cooperate with the DER power dispatch in such circumstances. So load shedding and DER response are integrated for frequency restoration. For simplicity, corresponding microgrid states are calculated based on steady-state characteristics.

In an island mode, the load supplied by microgrid DERs will increase when the utility supply is interrupted, resulting in the frequency drop. As we introduce load shedding in the microgrid, the system frequency will be restored to the quantity before islanding occurred. According to droop characteristics, the range of DER frequency regulation is determined by the DER droop coefficients and its operating state. Assuming that the DERs are numbered according to the descending range of their frequency regulations:

$$
m_{p, 1} \Delta P_{\max , 1} \geq m_{p, 2} \Delta P_{\max , 2} \geq \ldots \geq m_{p, n_{G}} \Delta P_{\max , n_{G}}
$$

where $m_{p, 1}$ is the active power droop coefficient of DER1, and $\Delta P_{\text {max, }}$ is the maximum power regulation capacity of DER1, and $n_{G}$ is the number of DERs. Fig. 1 shows the aggregated droop characteristics of microgrid DERs, in which the microgrid generation response in each segment to the frequency deviation is expressed as:

$$
\Delta P_{k}=\sum_{i=1}^{k} \frac{1}{m_{p, i}} \cdot \Delta f_{k}
$$

where $\Delta P_{k}$ represents the incremental microgrid load supplied by DERs which results in a frequency drop from $f_{k+1}$ to $f_{k}$, in island mode; $\Delta f_{k}$ is the range of frequency regulation in segment $k$.

In Fig. 1, the restoration of the nominal microgrid frequency $f^{\prime}$ located in segment $k$ will determine the required amount of load curtailment. Accordingly, if the microgrid frequency has dropped to $f^{\prime \prime}$ due to islanding, and $f^{\prime \prime}$ and $f^{\prime}$ are located in segments $j$ and $k$, respectively, the load curtailment required for restoring the frequency to $f^{\prime}$ is given as:

$$
\Delta P_{\text {shed }}^{\text {gross }}=\frac{\left(f_{j+1}-f^{\prime \prime}\right)}{\Delta f_{j}} \Delta P_{j}+\frac{\left(f^{\prime}-f_{k}\right)}{\Delta f_{k}} \Delta P_{k}+\sum_{i=j+1}^{k-1} \Delta P_{i}
$$

If the power deficit after islanding is beyond the regulation range of the combined droop curve, the total demand will be larger than the total capacity of the DERs. Hence, DERs will operate at their maximum capacities as the frequency deviation reaches beyond the acceptable range. Under such circumstances, according to the initial power deficit $\Delta P_{\text {def }}$ determined by measuring the power flow from the PCC prior to islanding, the required amount of load curtailment to ensure the frequency can settle in $f_{\text {restored }}$ can be expressed as:

$$
\Delta P_{\text {shed }}^{\text {gross }}=\Delta P_{\text {def }}-\left(\sum_{i=k+1}^{n} \Delta P_{i}+\frac{f_{k+1}-f^{\prime}}{\Delta f_{k}} \Delta P_{k}\right)
$$

Considering network losses, a loss factor $\alpha$, which is the ratio of initial load to initial generation, is introduced where:

$$
\Delta P_{\text {shed }}=(1-\alpha) \Delta P_{\text {shed }}^{\text {gross }}
$$

Owing to the fast response of inverter-based DERs, it is feasible to use the steady state frequency drop and restoration characteristics to determine load shedding. In this way, curtailment after islanding will be in line with the primary frequency control of DERs for frequency regulation.

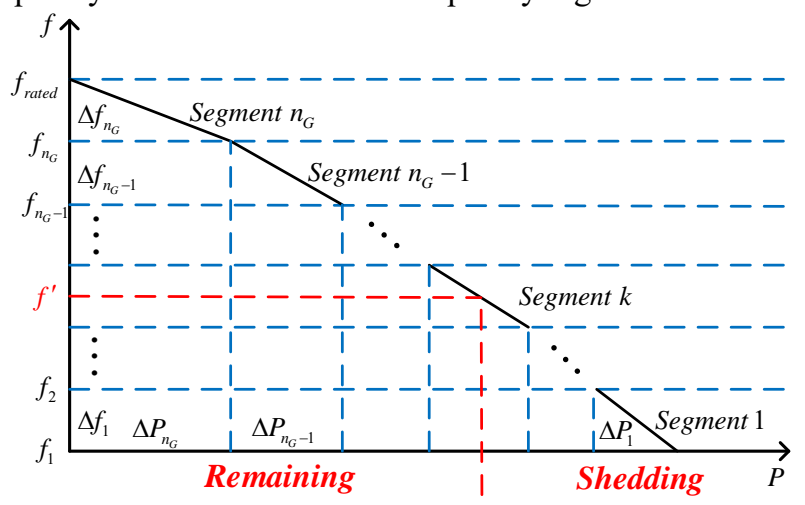

Fig. 1 Aggregated droop characteristic in a microgrid.

\section{POWER DEFICIT AND LOCAL FREQUENCY DEVIATIONS FOR INVERTER-BASED DERS}

As shown in Fig.2, the primary control structure for an inverter-based DER includes: (i) power sharing control (lower part of Fig.2), (ii) inverter output control (upper part of Fig.2). The power sharing control is to share active and reactive power demands among DERs according to their droop setting. The inverter output control, which consists of an outer loop for voltage control and an inner loop for current control, is aimed at regulating the output voltage and current of the inverter (DER voltage $V_{o}$ and current $I_{o}$ ) [26].

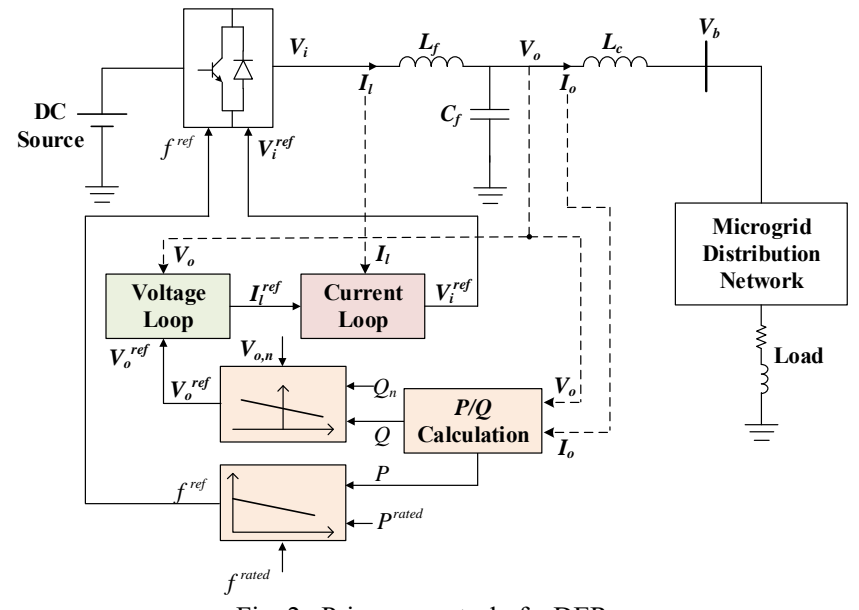

Fig. 2. Primary control of a DER. 
The droop control equations of DER $i$ is expressed as:

$$
\begin{aligned}
f_{i n v, i} & =f_{i n v, i}^{\text {rated }}-m_{P, i} \cdot\left(P_{i n v, i}-P_{i n v, i}^{\text {rated }}\right) \\
V_{i n v, i} & =V_{i n v, i}^{\text {rated }}-n_{Q, i} \cdot\left(Q_{i v v, i}-Q_{i v v, i}^{\text {rated }}\right)
\end{aligned}
$$

where $m_{P, i}$ and $n_{Q, i}$ are droop coefficients of active and reactive power for DER $i$, respectively, $f_{i n v, i}$ and $V_{i n v, i}$ are the frequency and voltage of DER $i$, respectively, $f_{i n v, i}^{\text {rated }}$ and $V_{i n v, i}^{\text {rated }}$ are the rated frequency and voltage of DER $i$, respectively, $P_{i n v, i}$ and $Q_{i n v, i}$ are the active and reactive power output of DER $i$, respectively, $P_{i n v, i}^{\text {rated }}$ and $Q_{i n v, i}^{\text {rated }}$ are the rated active and reactive power output of DER $i$, respectively.

The instantaneous active $p_{i n v, i}$ and reactive power output $q_{\text {ivv }, i}$ are calculated in the power sharing control by measuring output voltage and output current, i.e.,

$$
\begin{aligned}
& p_{\text {inv }, i}=V_{\text {invd }, i} \cdot I_{\text {invd }, i}+V_{\text {inv }, i} \cdot I_{\text {invq }, i} \\
& q_{\text {inv }, i}=V_{\text {inv }, i} \cdot I_{\text {invd }, i}-V_{\text {invd }, i} \cdot I_{\text {inv }, i}
\end{aligned}
$$

where $V_{\text {invd }, i}$ and $V_{\text {inv }, i}$ are the d-axis and q-axis components of output voltage of DER $i, I_{\text {invd }, i}$ and $I_{\text {invq }, i}$ are the d-axis and q-axis components of the output current of DER $i$.

To eliminate high-frequency harmonics, the instantaneous active and reactive power are passed by a low-pass filter, i.e.,

$$
P_{i n v, i}=\frac{f_{c}}{s+f_{c}} p_{i n v, i}, \quad Q_{i n v, i}=\frac{f_{c}}{s+f_{c}} q_{i n v, i}
$$

where $f_{c}$ is the cut-off frequency of the low-pass filter.

Equivalently,

$$
\begin{aligned}
& \frac{d P_{i n v, i}}{d t}+f_{c} P_{i n v, i}=f_{c}\left(V_{i n v d, i} \cdot I_{i n v d, i}+V_{i n v q, i} \cdot I_{i n v q, i}\right) \\
& \frac{d Q_{i n v, i}}{d t}+f_{c} Q_{i n v, i}=f_{c}\left(V_{i n v q, i} \cdot I_{i n v d, i}-V_{i n v d, i} \cdot I_{i n v q, i}\right)
\end{aligned}
$$

Then the relationship between frequency derivative and local power deficit can be formed as (similar to swing equations):

$$
\frac{1}{m_{P, i} f_{c}} \frac{d f_{i n v, i}}{d t}=P_{i n v, i}-P_{e, i}=\Delta P_{d e f, i}
$$

where $P_{i n v, i}$ is the active power output of DER $i$, and $P_{e, i}$ is the instantaneous power demand from the distribution network, defined as below:

$$
P_{e, i}=V_{\text {invd }, i} \cdot I_{\text {invd }, i}+V_{\text {invq }, i} \cdot I_{\text {inva }, i}
$$

If a power deficit occurs due to a sudden change in the microgrid operating condition (e.g. islanding, generator unit tripping, load change, etc.), the power deficit will be distributed among the participating DERs. Accordingly, the magnitude of power deficit at different microgrid locations will depend largely on the response of individual DERs. The transient response of DER power will depend on the microgrid damping conditions. Under such circumstances, the microgrid frequency derivative is an instantaneous indicator of local power deficit, while it is very sensitive to the pertinent transient, making the corresponding measurement rather cumbersome [9].

When facing a power deficit, the transient frequency decline rates are typically higher than $10 \mathrm{~Hz} / \mathrm{s}$ due to the low system inertia. Accordingly, there is not enough time to establish frequency drop rate and update the power deficit during the load shedding process. Although using the index of frequency derivative may improve the performance of load shedding scheme, the calculation of index may not be suitable for implementation in microgrids with low system inertia due to the oscillatory nature of the index and relative long delay in its execution.

After islanding, the local power deficit keeps change due to the response of DERs. By taking the integral of the local power deficit using an appropriate time constant, duration of the local power deficit is also taken into account. Then the relationship between local frequency deviation and the integral is formed as:

$$
\Delta f_{i}=m_{P, i} f_{c} \cdot \int \Delta P_{\text {def }, i}(t) d t
$$

where $\Delta f_{i}$ is the local frequency deviation, and $\int \Delta P_{\text {def }, i}(t) d t$ is the integral of local power deficit for a duration of time.

Hence, the local frequency deviation will distinguish the microgrid locations with local power deficits. The frequency difference among microgrid locations will continue until a new steady state frequency is reached. What is more, unlike the oscillatory frequency derivative, the frequency deviation and the integral of local power deficit will shrink after islanding. This trend is revealed in our simulation studies. Therefore, local frequency deviations and their relative differences can facilitate load shedding decisions in the low inertia microgrids.

\section{Proposed Two-stage Load Shedding Scheme}

In case of load shedding, the curtailment should take place appropriately along the designed load shedding steps. The sufficient curtailment should occur within a short period to cease the rapid frequency decline and prevent the frequency collapse. It is possible to drop smaller load quantities within the first steps to avoid any excessive load shedding. Even though the frequency collapse can be averted, operating at a low frequency may still pose a threat to the stability of microgrid. The microgrid will be vulnerable to additional disturbances in such occasions because the key microgrid parameters are designed using the rated operating states. Furthermore, if the frequency falls below a set point beyond a time limit, some DERs will cease to energize the microgrid resulting in additional power deficit. The frequency set point defined as a safe frequency $f_{\text {safe }}$ is field adjustable. Therefore, the load shedding should be sufficient to recover the frequency to the safe value within the time limit for preventing undesirable generator tripping in microgrids [27]. Then the secondary control, which is followed and implemented in the span of seconds or minutes, restores the rated microgrid frequency.

The proposed two-stage load shedding scheme addresses the power imbalance caused by microgrid islanding. The first stage is to cease rapid frequency drop as quickly as possible which allows the frequency to settle at a temporary settling point. Further load shedding is triggered at the second stage to recover the system frequency to a predetermined safe value. In Fig. 3, the microgrid frequency will deviate from its rated Point $\mathrm{A}$ and settle in Point B as load shedding occurs in island mode. At times, the frequency may continue to fall below Point B and rebound due to the slow response of certain DERs, like hydro turbines [28]. Within a time limit, additional load shedding is activated to recover the frequency to a predetermined Point C. At this time, the secondary control is initiated to restore the rated frequency at Point $\mathrm{D}$. 
The proposed flowchart is depicted in Fig. 4 and the steps are further analyzed as follows.

\section{A. First Stage of Load Shedding Scheme}

At the first stage shown in Fig. 4, the objective is to rescue the microgrid facing severe power deficit caused by islanding. To avoid too many loads being involved in this shedding stage, these loads should be selected regarding system stability rather than economics. Hence, the locally measured frequencies are utilized to distinguish the most effective loads after islanding for quickly arresting and stabilizing the system frequency.

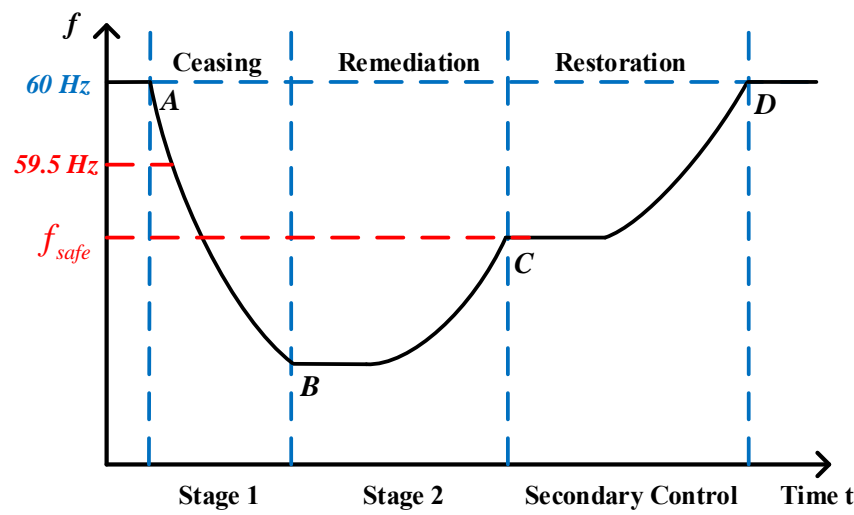

Fig. 3. Frequency response during load shedding process.

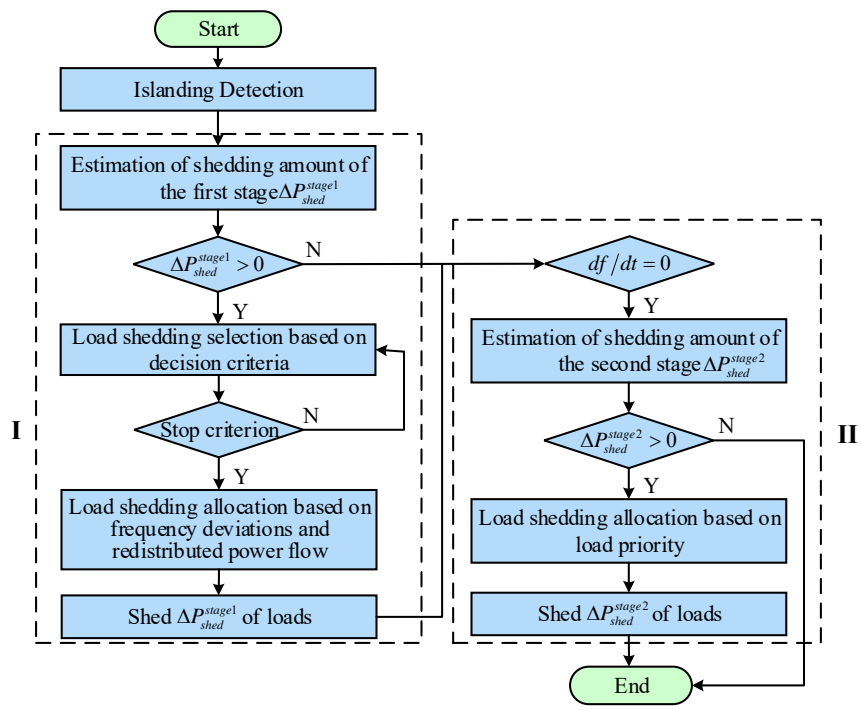

Fig. 4. Flowchart of the secondary control with two-stage load shedding.

We consider the following steps in order to optimize the quantity and the location of load shedding at the first stage:

\section{1) Load Shedding Quantity at the First Stage}

According to the first stage objective, load shedding can be divided into two parts:

a) Direct part: Eliminates the mismatch between the available power generation and load, namely,

$$
\Delta P_{\text {mis }}=\sum_{j=1}^{n_{D}} P_{D, j}-\sum_{i=1}^{n_{G}} P_{G, i}^{\max }
$$

where $n_{D}$ is the number of loads, $P_{D, j}$ is the demand of load $j$, and $P_{G, i}^{\max }$ is the maximum capacity of DER $i$. This part may fail to provide a sufficient frequency support.

b) Marginal part: Restores a temporary frequency (i.e., Point B in Fig. 3) by providing an adequate margin for frequency regulation which allows the restoration of rated frequency. The corresponding load curtailment is determined by (3).

2) Selection of Loads for Curtailment at the First Stage

The first stage will rescue the microgrid frequency in islanding mode. So, it is essential to identify a few loads in order to reduce the time delay for load shedding, especially in large microgrids with a large number of loads. In addition, the selection must exclude critical loads that ought to remain in operation and their curtailment in islanded microgrid may have detrimental effects on frequency or threaten the microgrid stability [28]. Therefore, the load shedding scheme must select the most effective loads to prevent system collapse.

As discussed in Section III, differences between local frequencies can reveal the severity of local power deficits. So they can be utilized to select specific loads for curtailment. Before islanding, local microgrid frequencies are all at the rated $60 \mathrm{~Hz}$. After islanding, the local frequencies drop towards a given threshold value. The threshold used in this article is $59.5 \mathrm{~Hz}$, which is shown in Fig. 3. Once the local frequency falls below $59.5 \mathrm{~Hz}$, the corresponding load is selected for curtailment. The stopping criterion for the selection is given as:

$$
\sum_{i \in S} P_{D, j}^{\text {net }} \geq \Delta P_{\text {shed }}^{\text {stagel }}
$$

where $S$ is the set of selected loads, $P_{D, j}^{\text {net }}$ is the net power injection at load bus $j$, and $\Delta P_{\text {shed }}^{\text {stagel }}$ is the estimated load shedding at the first stage. Once the stopping criterion is satisfied, the selection process will stop.

It is worth noting that these locations are not necessarily close to the location of the microgrid disturbance. Actually, they are usually located in areas where local DERs are insufficient for supplying the local demand. When facing a severe power deficit, a higher frequency threshold will be set so that the load shedding scheme can be triggered more quickly.

\section{3) Load Shedding Allocation at the First Stage}

After the load selection, the exact curtailment of each load is determined using frequency deviations and the estimated redistributed power flow in the microgrid, which is different from the initial load or power flow tracing based allocation [24]. Accordingly, a load bus with larger frequency deviation and heavier net load will have a larger share of curtailment.

Some DERs located close to load buses can respond quickly in order to carry the loads. After islanding, shedding any loads at such buses may lead to additional frequency fluctuations. Hence, local frequency deviations together with redistributed power flows will determine the load shedding allocation as:

$$
\Delta P_{\text {shed }, j}^{\text {stage }}=\frac{\Delta f_{D, j} \cdot P_{D, j}^{\text {net }}}{\sum_{j=1}^{n_{s}} \Delta f_{D, j} \cdot P_{D, j}^{\text {net }}} \cdot \Delta P_{\text {shed }}^{\text {stagel }}
$$

where $\Delta P_{\text {shed }, j}^{\text {stage }}$ is the curtailment at load $j$ in the first stage, $P_{D, j}^{\text {net }}$ is the net power injection at load bus $j$ after islanding, and $\Delta f_{D, j}$ is the locally measured frequency of load $j$. This stage of load shedding must be implemented quickly after islanding. Otherwise, severe power imbalances will lead to severe microgrid transients and even the frequency collapse.

\section{B. Second Stage of Load Shedding Scheme}

For avoiding undesirable generator tripping, the second stage 
of load shedding will recover the microgrid frequency from its temporary setting to the safe value (i.e., shifting from Point B to Point $\mathrm{C}$ in Fig. 3) which will be followed by secondary control. Through shifting each DER's operating point, the secondary control mitigates frequency and voltage deviations caused by the primary droop-based control. However, the process may introduce less-damped modes into the system, leading to oscillatory responses [29],[30]. Therefore, the load shedding in stage two is also considered to provide an adequate margin for frequency stability (Point $\mathrm{C}$ in Fig. 3). The additional curtailment will be calculated according to the steady state frequency drop and recovery characteristics.

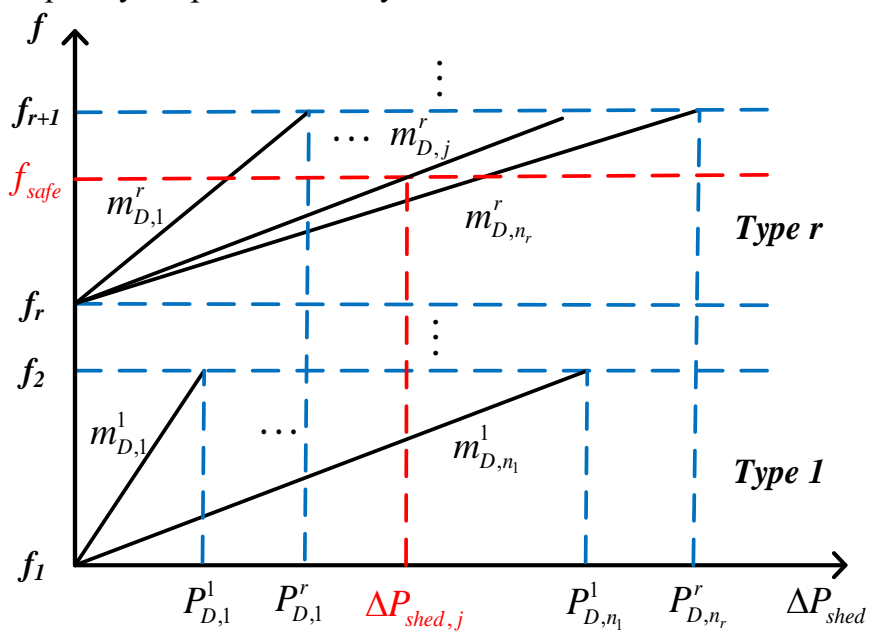

Fig. 5. Frequency recovery corresponding to load shedding levels.

Comparatively, the issue of delay time is not so critical in the second stage. Hence, the objective of this stage is not to rescue the system facing severe disturbance, but to find the most economic locations or reduce the total curtailment in the microgrid. At the second stage, the remaining loads are classified into several types according to their priorities. The loads with the lowest priority are assigned to the first type, which should be curtailed first.

The second stage is designed to recover the frequency from the temporary settling frequency $f_{1}$ to the safe value $f_{\text {safe }}\left(f_{r} \leq f_{\text {safe }} \leq f_{r+1}\right)$. As shown in Fig. 5, the loads designated as Type 1 to Type $r-1$ will be curtailed, which will shift the microgrid frequency from $f_{1}$ to $f_{r}$. Furthermore, additional curtailments are required to increase the frequency from $f_{r}$ to $f_{\text {safe }}$. This part should be proportionally shared among the loads of Type $r$, which consists of $n_{r}$ loads. So the recovery gain of load $j$ is defined as:

$$
m_{D, j}^{r}=\left(f_{r+1}-f_{r}\right) / P_{D, j}^{r}
$$

where $1 \leq j \leq n_{r}$ and $P_{D, j}^{r}$ is the initial demand of load $j$ of Type $r$. According to (19), the curtailment of each load of Type $r$ can be expressed as:

$$
\Delta P_{\text {shed }, j}=\left(f_{\text {safe }}-f_{r}\right) / m_{D, j}^{r}
$$

Accordingly, the required curtailment is obtained as:

$$
\Delta P_{\text {shed }}^{\text {stage } 2}=\sum_{l=1}^{r-1} P_{D}^{l}+\frac{f_{\text {safe }}-f_{r}}{f_{r+1}-f_{r}} \cdot P_{D}^{r}
$$

where $P_{D}^{r}$ is the total initial demand of Type $r$.
At this stage, load curtailment is determined based on priorities of individual loads, which ensure loads with low priority are curtailed first and critical loads are preserved.

\section{Case Studies}

\section{A. Studied Microgrid System}

As is shown in Fig. 6, the studied microgrid is a scaled-down three-phase balanced microgrid system with five DERs and six aggregate loads [29]. DER 1 to DER 4 are operated as interactive grid-forming DERs whereas DER 5 is operated as non-interactive grid-following DER. The microgrid is connected to the utility grid at the PCC through a circuit breaker, representing a common microgrid structure. The detailed technical parameters of the studied microgrid are shown in Table I. Since all the DERs are larger or equal to $30 \mathrm{~kW}$ in peak capacity, the safe frequency is set as $59 \mathrm{~Hz}$ and the corresponding time limit is 5s [27].

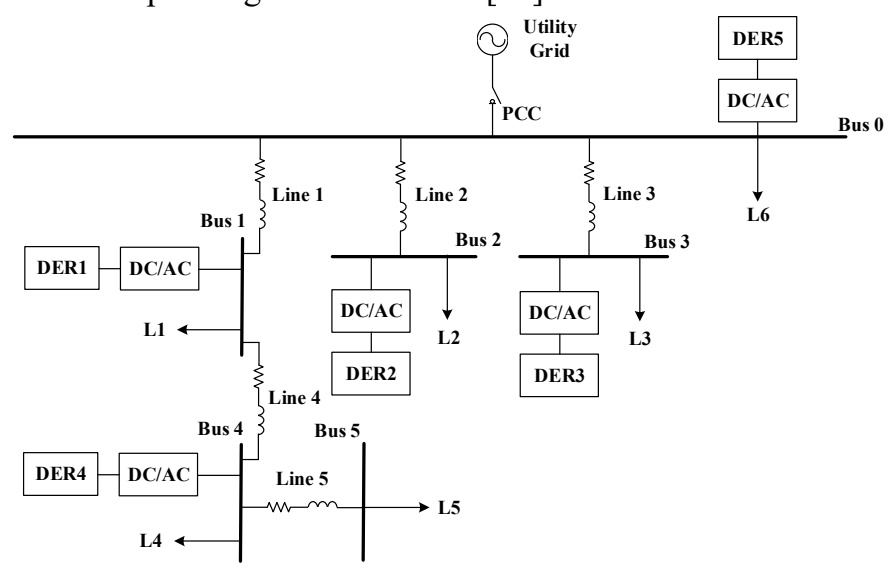

\begin{tabular}{|c|c|c|c|c|}
\hline \multicolumn{5}{|c|}{ DER } \\
\hline Parameter & $m_{P}(\mathrm{~Hz} / \mathrm{W})$ & $n_{Q}(\mathrm{~V} / \mathrm{Var})$ & $P_{\text {rated }}(\mathrm{kW})$ & $P_{\max }(\mathrm{kW})$ \\
\hline DER 1 & $5 e-2$ & $1 \mathrm{e}-4$ & 10 & 30 \\
\hline DER 2 & $3.75 \mathrm{e}-2$ & $1 \mathrm{e}-4$ & 10 & 50 \\
\hline DER 3 & $2.5 \mathrm{e}-2$ & $1 \mathrm{e}-4$ & 10 & 90 \\
\hline DER 4 & $2.5 \mathrm{e}-2$ & $1 \mathrm{e}-4$ & 10 & 110 \\
\hline DER 5 & - & - & 60 & 60 \\
\hline \multicolumn{5}{|c|}{ Network } \\
\hline Parameter & \multicolumn{2}{|c|}{ Resistance $\mathrm{R}(\Omega)$} & \multicolumn{2}{|c|}{ Reactance $\mathrm{X}(\mathrm{mH})$} \\
\hline Line $1,2,3$ & \multicolumn{2}{|c|}{0.1} & \multicolumn{2}{|c|}{0.5} \\
\hline Line 4,5 & \multicolumn{2}{|c|}{0.05} & \multicolumn{2}{|c|}{0.25} \\
\hline \multicolumn{5}{|c|}{ Loads } \\
\hline Parameter & \multicolumn{2}{|c|}{ Priority } & \multicolumn{2}{|c|}{ Active Power (kW) } \\
\hline Load 1 & \multicolumn{2}{|c|}{ Critical } & \multicolumn{2}{|c|}{60} \\
\hline Load 2 & \multicolumn{2}{|c|}{ Non-critical } & \multicolumn{2}{|c|}{80} \\
\hline Load 3 & \multicolumn{2}{|c|}{ Non- critical } & \multicolumn{2}{|c|}{80} \\
\hline Load 4 & \multicolumn{2}{|c|}{ Semi- critical } & \multicolumn{2}{|c|}{20} \\
\hline Load 5 & \multicolumn{2}{|c|}{ Semi- critical } & \multicolumn{2}{|c|}{20} \\
\hline Load 6 & \multicolumn{2}{|c|}{ Semi- critical } & \multicolumn{2}{|c|}{100} \\
\hline
\end{tabular}

Fig. 6. A schematic diagram of the studied microgrid system

Table I Technical specifications of the studied microgrid

The total delay for activating relays and load shedding is $50 \mathrm{~ms}$ ( 3 cycles). The communication delay and calculation time required by microgrid master controller will take $50 \mathrm{~ms}$ at the first stage. For comparison, we consider a UFLS scheme with a 4-step load shedding of $35-30-20-15 \%$ at threshold frequencies, 59, 58.8, 58.4 and $58 \mathrm{~Hz}$. These frequency thresholds and load shedding allocation in each step are deliberately chosen to minimize the load curtailment for higher levels of power deficit [17]. 
We consider the following assumptions for simulation:

1) The grid-following DERs are WTGs, and grid-forming DERs are a combination of WTGs and BESS [31];

2) Grid-following DERs are modeled as PQ-controlled voltage source converters for maintaining pre-determined active and reactive power outputs;

3) Grid-forming DERs are modeled as droop-controlled voltage source converters, which interact with other DERs for sharing load demands autonomously;

4) DERs are connected to the microgrid distribution network through an identical LCL filter;

5) Loads are controllable. If a load is curtailed, its active and reactive power consumptions (determined by the load power factor) will be interrupted;

6) For improving the system dynamic performance, part of L6, which is directly supplied by a grid-following DER, does not participate in load shedding unless this DER is tripped.

The proposed load shedding scheme is tested in the PSCAD/EMTDC platform using this microgrid system.

\section{B. Simulation Cases}

Three simulation cases are carried out:

Case 1: Microgrid is islanded from the utility grid;

Case 2: Microgrid islanding and lower load demand;

Case 3: Microgrid islanding and outage of DER;

The microgrid islanding is simulated by opening the circuit breaker at $\mathrm{t}=1 \mathrm{~s}$. The simulation is recorded for $15 \mathrm{~s}$.

\section{Case 1: Microgrid Islanded from the Utility Grid}

Before islanding, the total load is $360 \mathrm{~kW}$ and the microgrid network loss is $15 \mathrm{~kW}$. The supply from the utility grid and DERs are $275 \mathrm{~kW}$ and $100 \mathrm{~kW}$, respectively. So the loss factor is $4.2 \%$. When islanding occurs, the interruption of utility supply will cause severe active power deficit. The DERs increase their supplies in the islanded microgrid. However, the power deficit will continue due to the limited DER capacity.

The load curtailment at the first stage is determined by (4) and (5) as $52.8 \mathrm{~kW}$. According to the locally measured frequencies, L6 and L2 are selected successively to participate at the first stage load shedding (LS) with the corresponding allocation determined by (18). Load shedding provides sufficient support for system frequency, which is ceased at $58 \mathrm{~Hz}$. At $\mathrm{t}=3 \mathrm{~s}$, the second stage load shedding is activated and shared by L2 and L3 due to their lower priorities. The corresponding curtailment is calculated by (21) as $89.6 \mathrm{~kW}$. Accordingly, the frequency is recovered to the safe value $59 \mathrm{~Hz}$. At $\mathrm{t}=10 \mathrm{~s}$, the secondary control is initiated which restores the rated frequency $60 \mathrm{~Hz}$. The microgrid frequency response and active power adjustment are depicted in Figs. 7 and 8, respectively.

For the 4-step UFLS scheme, the first three steps of load shedding are triggered at $\mathrm{t}=1.22 \mathrm{~s}, 1.25 \mathrm{~s}$ and $1.35 \mathrm{~s}$, respectively. In the first step, only L2 and L3 are selected due to their low priorities. Then all the other loads, except for L1, will be involved in this load shedding scheme. Fig. 9 shows the comparison of the system frequency response in the two schemes, with the corresponding information on activated load shedding for each scheme presented in Table II. Clearly, there is a delay of the frequency support provided by load shedding. Both schemes have limited the frequency deviation after islanding event to $58 \mathrm{~Hz}$. However, the level of curtailment and the number of involved loads are much fewer in the proposed scheme.

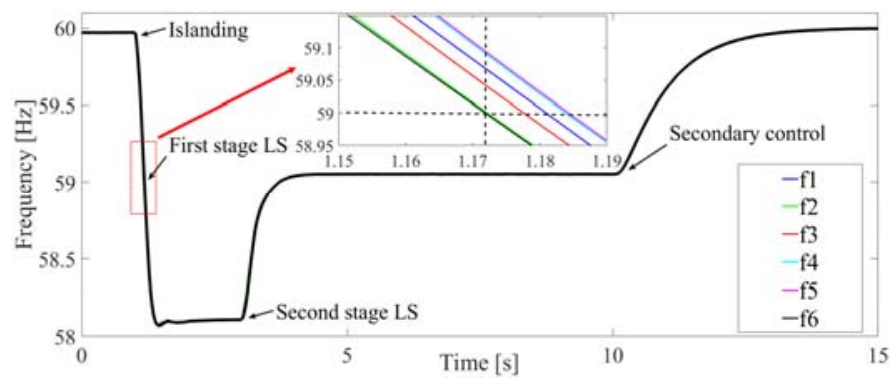

Fig. 7. Frequency response using the proposed LS scheme in Case 1

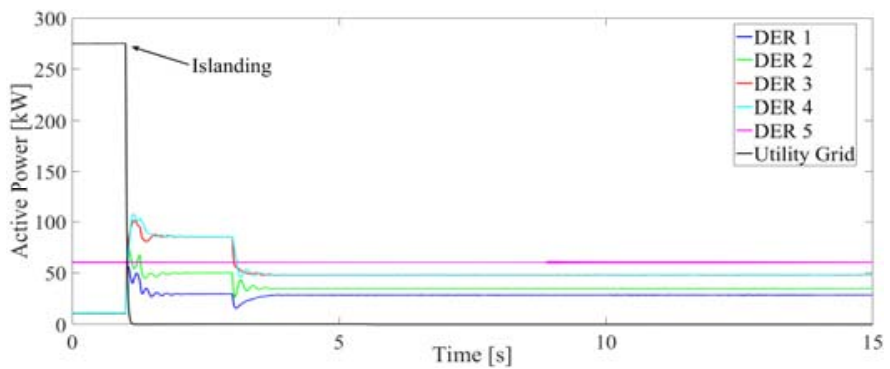

Fig. 8. Active power of DERs and utility grid in Case 1

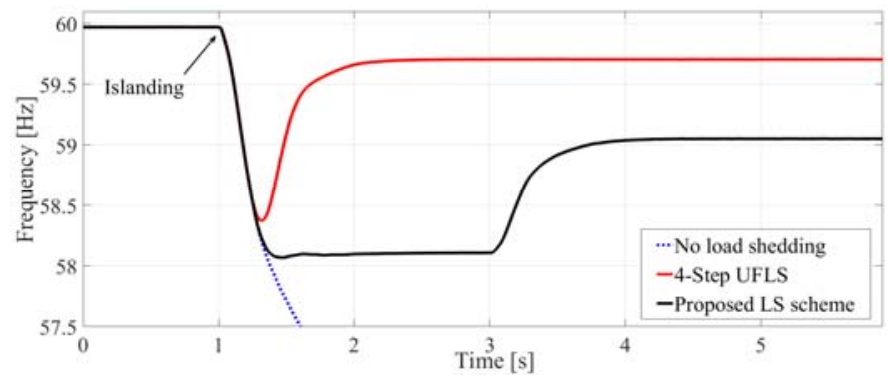

Fig. 9. Comparison of system frequency response in Case 1

Table II Load shedding from each load in the two schemes $(\mathrm{kW})$

\begin{tabular}{|c|c|c|c|c|c|c|c|c|}
\hline \multicolumn{9}{|c|}{ Proposed Load Shedding Scheme } \\
\hline \multirow[t]{2}{*}{ Stage } & \multirow{2}{*}{$\begin{array}{c}\text { Activation } \\
\text { Time (s) }\end{array}$} & \multicolumn{6}{|c|}{$\begin{array}{l}\text { Load }(\mathrm{kW}) \\
\end{array}$} & \multirow[b]{2}{*}{ Sum } \\
\hline & & L1 & L2 & L3 & L4 & L5 & L6 & \\
\hline 1 & 1.27 & 0 & 22.63 & 0 & 0 & 0 & 30.17 & 52.8 \\
\hline 2 & 3.00 & 0 & 37.42 & 52.18 & 0 & 0 & 0 & 89.6 \\
\hline \multicolumn{2}{|c|}{ Load Shedding $(\mathrm{kW})$} & 0 & 60.05 & 52.18 & 0 & 0 & 30.17 & 142.4 \\
\hline \multicolumn{9}{|c|}{ Conventional 4-Step UFLS Scheme } \\
\hline \multirow[t]{2}{*}{ Step } & \multirow{2}{*}{$\begin{array}{c}\text { Activation } \\
\text { Time (s) }\end{array}$} & \multicolumn{6}{|c|}{ Load $(\mathrm{kW})$} & \\
\hline & & L1 & $\mathbf{L 2}$ & $\mathbf{L 3}$ & L4 & L5 & L6 & Sum \\
\hline 1 & 1.22 & 0 & 46.2 & 46.2 & 0 & 0 & 0 & 92.4 \\
\hline 2 & 1.25 & 0 & 33.8 & 33.8 & 2.9 & 2.9 & 5.8 & 79.2 \\
\hline 3 & 1.35 & 0 & 0 & 0 & 13.2 & 13.2 & 26.4 & 52.8 \\
\hline 4 & - & 0 & 0 & 0 & 0 & 0 & 0 & 0 \\
\hline \multicolumn{2}{|c|}{ Load Shedding $(\mathrm{kW})$} & 0 & 80 & 80 & 16.1 & 16.1 & 32.2 & 224.4 \\
\hline
\end{tabular}

\section{Case 2: Islanding with Lower Load Demand}

In this case, the islanding and partial outage of $\mathrm{L} 3$ by $60 \mathrm{~kW}$ occur simultaneously. The frequency response for the proposed scheme is depicted in Fig. 10. The change of power deficit is successfully recognized by the proposed scheme. Considering (4) and (5), there are no loads to be curtailed at the first stage. At $\mathrm{t}=3 \mathrm{~s}$, the new steady state is reached and the second stage will curtail L2 and L3 by $65.92 \mathrm{~kW}$ and $16.48 \mathrm{~kW}$, respectively, as determined by (21). Since the curtailment at stage one is zero, the curtailment of each load is only decided by their priorities.

For this case, only two load shedding steps are triggered in the 4-step scheme due to the lower initial power deficit. The curtailment is reduced to $134.16 \mathrm{~kW}$. But there are still five loads involved in the load shedding procedure. Table III 
presents the curtailment of each load in each scheme. In Fig. 11, the frequency performance of the 4-step scheme is better than that in Case 1. When facing lower power deficits, the proposed scheme still has a better performance than that of the 4-step scheme regarding the level of curtailment and the number of involved loads.

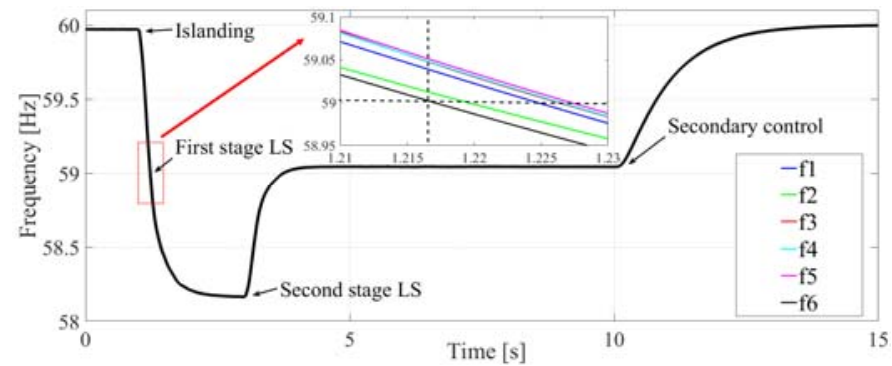

Fig. 10. Frequency response using the proposed LS scheme in Case 2

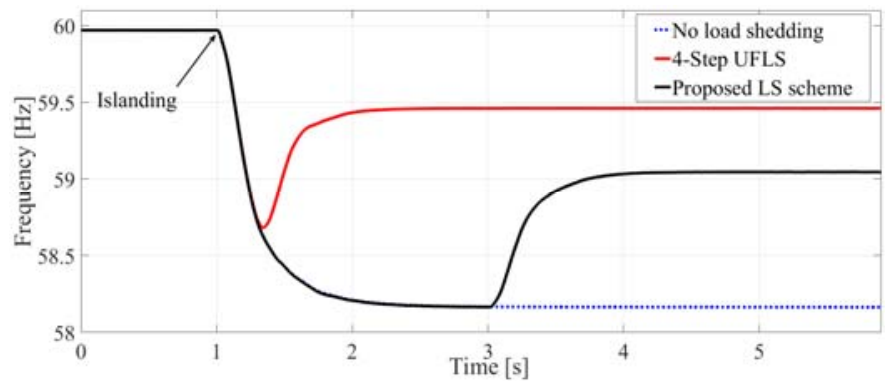

Fig. 11. Comparison of system frequency response in Case 2

Table III Load shedding from each load in the two schemes $(\mathrm{kW})$

\begin{tabular}{|c|c|c|c|c|c|c|c|c|}
\hline \multicolumn{9}{|c|}{ Proposed Load Shedding Scheme } \\
\hline \multirow[t]{2}{*}{ Stage } & \multirow{2}{*}{$\begin{array}{c}\text { Activation } \\
\text { Time (s) }\end{array}$} & \multicolumn{6}{|c|}{ Load } & \multirow[b]{2}{*}{ Sum } \\
\hline & & L1 & $\mathbf{L 2}$ & L3 & L4 & L5 & L6 & \\
\hline 1 & 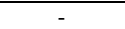 & 0 & 0 & 0 & 0 & 0 & 0 & 0 \\
\hline 2 & 3.00 & 0 & 65.92 & 16.48 & 0 & 0 & 0 & 82.40 \\
\hline \multicolumn{2}{|c|}{ Load Shedding } & 0 & 65.92 & 16.48 & 0 & 0 & 0 & 82.40 \\
\hline \multicolumn{9}{|c|}{ Conventional 4-Step UFLS Scheme } \\
\hline \multirow[t]{2}{*}{ Step } & Activation & \multicolumn{6}{|c|}{ Load } & \\
\hline & Time (s) & L1 & $\mathbf{L 2}$ & $\mathbf{L 3}$ & $\mathbf{L 4}$ & L5 & L6 & Sum \\
\hline 1 & 1.27 & 0 & 57.79 & 14.45 & 0 & 0 & 0 & 72.24 \\
\hline 2 & 1.32 & 0 & 22.21 & 5.55 & 8.54 & 8.54 & 17.08 & 61.92 \\
\hline 3 & - & 0 & 0 & 0 & 0 & 0 & 0 & 0 \\
\hline 4 & - & 0 & 0 & 0 & 0 & 0 & 0 & 0 \\
\hline Load & Shedding & 0 & 80.00 & 20.00 & 8.54 & 8.54 & 17.08 & 134.16 \\
\hline
\end{tabular}

Case 3: Islanding with Outage of DER

The DER 5 is operated as a non-interactive grid-following WTG. However, if the wind speed exceeds beyond the cut-out value, this DER will be tripped to avoid damages to WTG.

In this case, we assume the power deficit is caused by the simultaneous microgrid islanding and the tripping of DER 5. Compared to Case 1, there is an additional power deficit resulted by the DER 5 outage. Accordingly, the active power responses of DERs and the utility gird are also changed as depicted in Fig. 12.

According to (4) and (5), the curtailment at stage one is $112.8 \mathrm{~kW}$ which is allocated among L2 and L6 and implemented at $\mathrm{t}=1.24 \mathrm{~s}$. The lower frequency is $57.5 \mathrm{~Hz}$ and the temporary frequency is set at $58 \mathrm{~Hz}$ which is reached at $\mathrm{t}=3 \mathrm{~s}$. At the second stage, the curtailment is $89.6 \mathrm{~kW}$, which is allocated among L2 and L3 according to their available capacities. At $\mathrm{t}=10 \mathrm{~s}$, the frequency is gradually restored by the subsequent secondary control. Fig. 13 depicts the frequency response of the microgrid.

A comparison of the proposed LS scheme with the 4-step UFLS scheme is shown in Fig. 14. Due to the additional power deficit, system frequency drops more quickly than that in Case
1. Although larger curtailment has occurred during the first few steps, the frequency still falls below $58 \mathrm{~Hz}$, resulting all the four load shedding steps are triggered. Even the critical load L1 has been forced to shed part of its load demand. The excessive load shedding brings the system frequency above $60 \mathrm{~Hz}$. The curtailment at each scheme is represented in Table IV. When facing a higher power deficit, the 4-step UFLS may result in serious over-load-shedding. Comparatively, the proposed scheme can successfully rescue the frequency drop with a smaller level of curtailment.

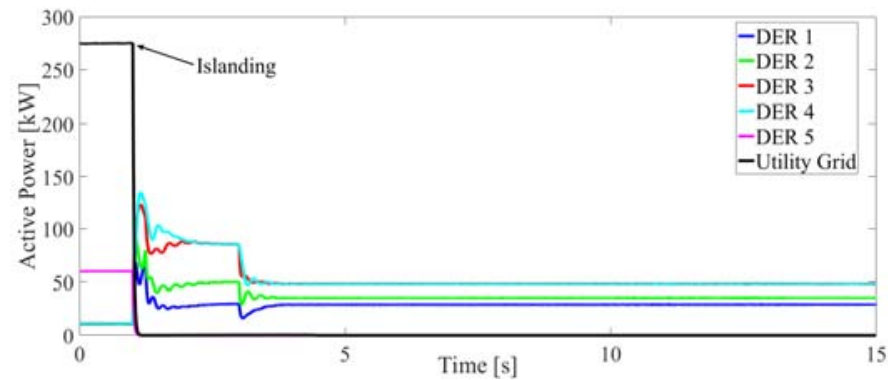

Fig. 12. Active power of DERs and utility grid in Case 3

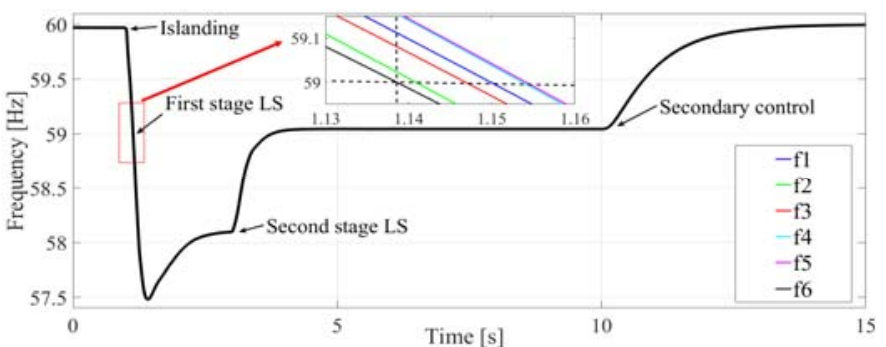

Fig. 13. Frequency response using the proposed LS scheme in Case 3

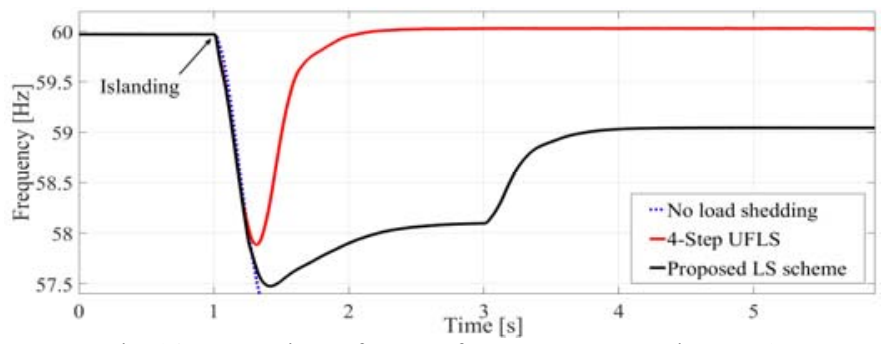

Fig. 14. Comparison of system frequency response in Case 3

Table IV Load shedding from each load in the two schemes (kW)

\begin{tabular}{|c|c|c|c|c|c|c|c|c|}
\hline \multicolumn{9}{|c|}{ Proposed Load Shedding Scheme } \\
\hline \multirow[t]{2}{*}{ Stage } & \multirow{2}{*}{$\begin{array}{c}\text { Activation } \\
\text { Time (s) }\end{array}$} & \multicolumn{6}{|c|}{ Load } & \multirow[b]{2}{*}{ Sum } \\
\hline & & L1 & L2 & L3 & L4 & L5 & L6 & \\
\hline 1 & 1.24 & 0 & 26.03 & 0 & 0 & 0 & 86.77 & 112.80 \\
\hline 2 & 3.00 & 0 & 36.10 & 53.50 & 0 & 0 & 0 & 89.60 \\
\hline \multicolumn{2}{|c|}{ Load Shedding } & 0 & 62.13 & 53.50 & 0 & 0 & 86.77 & 202.40 \\
\hline \multicolumn{9}{|c|}{ Conventional 4-Step UFLS Scheme } \\
\hline \multirow[t]{2}{*}{ Step } & Activation & \multicolumn{6}{|c|}{ Load } & \\
\hline & Time (s) & L1 & L2 & L3 & L4 & L5 & L6 & Sum \\
\hline 1 & 1.19 & 0 & 56.70 & 56.70 & 0 & 0 & 0 & 113.40 \\
\hline 2 & 1.21 & 0 & 23.30 & 23.30 & 7.23 & 7.23 & 36.14 & 97.20 \\
\hline 3 & 1.26 & 0 & 0 & 0 & 9.26 & 9.26 & 46.30 & 64.80 \\
\hline 4 & 1.32 & 24.03 & 0 & 0 & 3.51 & 3.51 & 17.56 & 48.6 \\
\hline \multicolumn{2}{|c|}{ Load Shedding } & 24.03 & 80.00 & 80.00 & 20.00 & 20.00 & 100.00 & 324.0 \\
\hline
\end{tabular}

In the three cases, load shedding levels at the conventional 4 -step UFLS scheme are $224.4 \mathrm{~kW}, 134.16 \mathrm{~kW}$, and $324 \mathrm{~kW}$, respectively. Due to the delay of the frequency recovery provided by load shedding, the rapidly increased frequency deviation may make too many steps to be triggered, resulting in excessive load shedding and even over frequency conditions. Clearly, there are limitations for UFLS schemes (triggered step by step) to deal with the rapid frequency drop in low inertia microgrids. 
Comparatively, the curtailments for the three cases by using the proposed load shedding scheme are significantly reduced as $142.4 \mathrm{~kW}, 82.4 \mathrm{~kW}$ and $202.4 \mathrm{~kW}$, respectively. It makes full use of secondary control by carefully tuning the amounts and the allocations of load curtailments. So the proposed solution can have a desirable frequency regulation performance for different levels of power deficit. It is worth noting that the number of involved loads is also reduced in the proposed approch to secondary control.

\section{CONCLUSION}

In order to cease the rapid frequency drop, load shedding scheme is usually designed in a "better more than less" manner, resulting in some loads are unnecessarily shed. That is because the system frequency must already fall below the threshold value before the corresponding load shedding can operate. What is more, there is a delay before the active power compensation provided by the load shedding is reflected in the system frequency response. Therefore, even with carefully adjusted parameters of load shedding, such as number of steps, frequency thresholds and curtailment of each step, the performance in frequency regulation may still be undesirable under different operating conditions. Especially for microgrids with low system inertia, the rapid frequency drop may easily trigger too many load shedding steps and then causes over load shedding, which is revealed in the simulation results.

This paper presents a two-stage load shedding scheme to address the severe under frequency conditions after islanding. The first stage is responsible for ceasing the rapid frequency drop and rescuing the system from collapse. Most effective loads are selected to be shed based on the locally measured information and the final power flow distribution. The second stage fulfills the steady state frequency recovery between two settling frequencies. With consideration of the fast primary frequency response of inverter-based DERs, the curtailment of each stage is carefully adjusted according to the actual system operating conditions. The time-domain simulation case studies have been presented to shown the robust performance of the proposed load shedding scheme in frequency regulation.

\section{REFERENCES}

[1] L. Che and M. Shahidehpour, "DC microgrids: economic operation and enhancement of resilience by hierarchical control," IEEE Trans. Smart Grid, vol. 5, no. 5, pp. 2517-2526, Sept. 2014.

[2] M. Shahidehpour and M. Khodayar, "Cutting campus energy costs with hierarchical control: The economical and reliable operation of a microgrid," IEEE Electrif. Mag., vol. 1, no. 1, pp. 40-56, Sep. 2013.

[3] A. Gholami, T. Shekari, F. Aminifar and M. Shahidehpour, "Microgrid scheduling with uncertainty: The quest for resilience," IEEE Trans. Smart Grid, vol. 7, no. 6, pp. 2849-2858, Nov. 2016.

[4] M. E. Khodayar, M. Barati and M. Shahidehpour, "Integration of high reliability distribution system in microgrid operation," IEEE Trans. Smart Grid, vol. 3, no. 4, pp. 1997-2006, Dec. 2012.

[5] A. Bidram and A. Davoudi, "Hierarchical structure of microgrids control system," IEEE Trans. Smart Grid, vol. 3, no. 4, pp. 1963-1976, Dec. 2012.

[6] X. Liu, M. Shahidehpour, Z. Li, X. Liu, Y. Cao and Z. Bie, "Microgrids for enhancing the power grid resilience in extreme conditions," IEEE Trans. Smart Grid, vol. 8, no. 2, pp. 589-597, March 2017.

[7] L. Che, M. Khodayar and M. Shahidehpour, "Only connect: Microgrids for distribution system restoration," IEEE Power Energy Mag., vol. 12, no. 1, pp. 70-81, Jan.-Feb. 2014.

[8] L. Che, M. Shahidehpour, A. Alabdulwahab and Y. Al-Turki, "Hierarchical coordination of a community microgrid with AC and DC microgrids," IEEE Trans. Smart Grid, vol. 6, no. 6, pp. 3042-3051, Nov. 2015.

[9] IEEE Guide for the Application of Protective Relays Used for Abnormal Frequency Load Shedding and Restoration, IEEE Std. C37. 117-2007, Aug. 2007.

[10] M. Karimi, P. Wall, H. Mokhlis and V. Terzija, "A new centralized adaptive underfrequency load shedding controller for microgrids based on a distribution state estimator," IEEE Trans. Power Del., vol. 32, no. 1, pp. 370-380, Feb. 2017

[11] A. Ketabi and M. H. Fini, "An underfrequency load shedding scheme for hybrid and multiarea power systems," IEEE Trans. Smart Grid, vol. 6, no. 1, pp. 82-91, Jan. 2015.

[12] Y. Y. Hong, M. C. Hsiao, Y. R. Chang, Y. D. Lee and H. C. Huang, "Multiscenario underfrequency load shedding in a microgrid consisting of intermittent renewables," IEEE Trans. Power Del., vol. 28, no. 3, pp. 1610-1617, July 2013.

[13] A. Saffarian and M. Sanaye-Pasand, "Enhancement of power system stability using adaptive combinational load shedding methods," IEEE Trans. Power Syst., vol. 26, no. 3, pp. 1010-1020, Aug. 2011.

[14] V. V. Terzija, "Adaptive underfrequency load shedding based on the magnitude of the disturbance estimation," IEEE Trans. Power Syst., vol. 21, no. 3, pp. 1260-1266, Aug. 2006.

[15] U. Rudez and R. Mihalic, "Analysis of underfrequency load shedding using a frequency gradient," IEEE Trans. Power Del., vol. 26, no. 2, pp. 565-575, April 2011.

[16] T. Shekari, F. Aminifar and M. Sanaye-Pasand, "An analytical adaptive load shedding scheme against severe combinational disturbances," IEEE Trans. Power Syst., vol. 31, no. 5, pp. 4135-4143, Sept. 2016.

[17] U. Rudez and R. Mihalic, "Monitoring the first frequency derivative to improve adaptive underfrequency load-shedding schemes," IEEE Trans. Power Syst., vol. 26, no. 2, pp. 839-846, May 2011.

[18] D. Ochoa; S. Martinez, "Fast-frequency response provided by DFIG-wind turbines and its impact on the grid," IEEE Trans. Power Syst., to be published. DOI: 10.1109/TPWRS.2016.2636374.

[19] L. Che, M. E. Khodayar and M. Shahidehpour, "Adaptive protection system for microgrids: Protection practices of a functional microgrid system," IEEE Electrif. Mag., vol. 2, no. 1, pp. 66-80, March 2014.

[20] M. E. Khodayar, M. Shahidehpour and L. Wu, "Enhancing the dispatchability of variable wind generation by coordination with pumped-storage hydro units in stochastic power systems," IEEE Trans. Power Syst., vol. 28, no. 3, pp. 2808-2818, Aug. 2013.

[21] NPCC Document A-03, Emergency Operation Criteria, Northeast Power Coordinating Council (NPCC), Aug. 31, 2004, p. 4.

[22] L. Tang and J. McCalley, "Two-stage load control for severe under-frequency conditions," IEEE Trans. Power Syst., vol. 31, no. 3, pp. 1943-1953, May 2016.

[23] Q. C. Zhong and G. Weiss, "Synchronverters: Inverters that mimic synchronous generators," IEEE Trans. Ind. Electron., vol. 58, no. 4, pp. 1259-1267, April 2011.

[24] J. Tang, J. Liu, F. Ponci and A. Monti, “Adaptive load shedding based on combined frequency and voltage stability assessment using synchrophasor measurements," IEEE Trans. Power Syst., vol. 28, no. 2, pp. 2035-2047, May 2013.

[25] E. Pashajavid and A. Ghosh, "Frequency support for remote microgrid systems with intermittent distributed energy resources-A two-level hierarchical strategy," IEEE Trans. Power J., to be published. DOI: 10.1109/JSYST.2017.2661743.

[26] N. Pogaku, M. Prodanović, and T. C. Green, "Modeling, analysis and testing of autonomous operation of an inverter-based microgrid," IEEE Trans. Power Electron., vol. 22, no. 2, pp. 613-625, 2007.

[27] IEEE Guide for Conducting Distribution Impact Studies for Distributed Resource Interconnection, IEEE Std. 1547.7-2013, Feb. 282014.

[28] D. Rodriguez Medina et al., "Fast assessment of frequency response of cold load pickup in power system restoration," IEEE Trans. Power Syst., vol. 31, no. 4, pp. 3249-3256, July 2016.

[29] X. Wu and C. Shen, "Distributed optimal control for stability enhancement of microgrids with multiple distributed generators," IEEE Trans. Power Syst., 2017.

[30] S. Falahati, S. A. Taher, M. Shahidehpour, "Grid secondary frequency control by optimized fuzzy control of electric vehicles," IEEE Trans. Power Syst., to be published. DOI: 10.1109/TSG.2017.2692265.

[31] Y. Sun, J. Zhong, Z. Li, W. Tian and M. Shahidehpour, "Stochastic scheduling of battery-based energy storage transportation system with the penetration of wind power," IEEE Trans. Sustain. Energy, vol. 8, no. 1, pp. 135-144, Jan. 2017. 